Publ. Mat. 63 (2019), 241-264

DOI: 10.5565 /PUBLMAT6311908

\title{
WEAK-2-LOCAL ISOMETRIES ON UNIFORM ALGEBRAS AND LIPSCHITZ ALGEBRAS
}

\author{
Lei Li, Antonio M. Peralta, Liguang Wang, \\ AND YA-SHU WANG
}

\begin{abstract}
We establish spherical variants of the Gleason-Kahane-Żelazko and Kowalski-Słodkowski theorems, and we apply them to prove that every weak-2-local isometry between two uniform algebras is a linear map. Among the consequences, we solve a couple of problems posed by O. Hatori, T. Miura, H. Oka, and H. Takagi in 2007.

Another application is given in the setting of weak-2-local isometries between Lipschitz algebras by showing that given two metric spaces $E$ and $F$ such that the set $\operatorname{Iso}((\operatorname{Lip}(E),\|\cdot\|),(\operatorname{Lip}(F),\|\cdot\|))$ is canonical, then every weak-2-local $\operatorname{Iso}((\operatorname{Lip}(E)$, $\|\cdot\|),(\operatorname{Lip}(F),\|\cdot\|))$-map $\Delta$ from $\operatorname{Lip}(E)$ to $\operatorname{Lip}(F)$ is a linear map, where $\|\cdot\|$ can indistinctly stand for $\|f\|_{L}:=\max \left\{L(f),\|f\|_{\infty}\right\}$ or $\|f\|_{s}:=L(f)+\|f\|_{\infty}$.
\end{abstract}

2010 Mathematics Subject Classification: Primary: 46B04, 46B20, 46J10, 46E15; Secondary: 30H05, 32A38, 46J15, 47B48, 47B38, $47 \mathrm{D} 03$.

Key words: 2-local isometries, uniform algebras, Lipschitz functions, spherical Gleason-Kahane-Żelazko theorem, spherical Kowalski-Słodkowski theorem, weak-2-local isometries.

\section{Introduction}

Let Iso $(X, Y)$ denote the set of all surjective linear isometries between two Banach spaces $X$ and $Y$. Clearly Iso $(X, Y)$ can be regarded as a subset of the space $L(X, Y)$ of all linear maps between $X$ and $Y$. We shall write Iso $(X)$ instead of $\operatorname{Iso}(X, X)$. Accordingly to the notation in $[\mathbf{1 2}, \mathbf{1 3}, \mathbf{3 9}, \mathbf{3 8}]$ and $[\mathbf{4 2}]$, we shall say that a (not-necessarily linear nor continuous) mapping $\Delta: X \rightarrow Y$ is a weak-2-local $\operatorname{Iso}(X, Y)$-map or a weak-2-local isometry (respectively, a 2-local Iso $(X, Y)$-map or a 2-local isometry) if for each $x, y \in X$ and $\phi \in Y^{*}$, there exists $T_{x, y, \phi}$ in Iso $(X, Y)$, depending on $x, y$, and $\phi$ (respectively, for each $x, y \in X$, there exists $T_{x, y}$ in Iso $(X, Y)$, depending on $x$ and $\left.y\right)$, satisfying

$$
\phi \Delta(x)=\phi T_{x, y, \phi}(x) \text { and } \phi \Delta(y)=\phi T_{x, y, \phi}(y)
$$

(respectively, $\Delta(x)=T_{x, y}(x)$ and $\Delta(y)=T_{x, y}(y)$ ). A Banach space $X$ is said to be (weak-2-iso-reflexive if every (weak-)2-local isometry on $X$ is both linear and surjective. 
If in the above definition $A$ and $B$ are Banach algebras and the set $\operatorname{Iso}(A, B)$ is replaced by the set $\mathcal{G}(A, B)$ of all algebra isomorphisms from $A$ onto $B$ we obtain the notion of (weak-)2-local isomorphism. For $A=B$ (weak-)2-local isomorphisms are called (weak-)2-local automorphisms.

For $1 \leq p<\infty$ and $p \neq 2$, Al-Halees and Fleming [1] showed that $\ell^{p}$ is 2-iso-reflexive. In the setting of $B(H), \mathrm{C}^{*}$-algebras and $\mathrm{JB}^{*}$-triples there exists a extensive literature on different classes of (weak-)2-local maps (see, for example, $[2,3,4,9,10,12,13,18,19,22,30,34,39,38]$ and $[42])$.

There are two main questions treated in recent times, the first asks whether every (weak-)2-local isometry between certain Banach spaces $X$ and $Y$ is a linear mapping. If the answer to the first one is affirmative, is every (weak-)2-local isometry between $X$ and $Y$ a surjective isometry?

Let $E$ and $F$ denote two metric spaces. A function $f: E \rightarrow F$ is called Lipschitz if its Lipschitz number

$$
L(f):=\sup \left\{\frac{d_{F}(f(x), f(y))}{d_{E}(x, y)}: x, y \in E, x \neq y\right\}
$$

is finite. When $F=Y$ is a $\operatorname{Banach}$ space, the $\operatorname{symbol} \operatorname{Lip}(E, Y)$ will denote the space of all bounded Lipschitz functions from $E$ into $Y$. The space $\operatorname{Lip}(E, Y)$ is a Banach space with respect to the following equivalent and complete norms

$$
\|f\|_{L}:=\max \left\{L(f),\|f\|_{\infty}\right\} \text { and }\|f\|_{s}:=L(f)+\|f\|_{\infty} .
$$

Throughout this note $\mathbb{F}$ will either stand for $\mathbb{R}$ or $\mathbb{C}$, and we shall write $\operatorname{Lip}(E)$ for the space $\operatorname{Lip}(E, \mathbb{C})$. It is known that, for every metric space $E,\left(\operatorname{Lip}(E),\|\cdot\|_{s}\right)$ is a unital commutative complex Banach algebra with respect to pointwise multiplication (see [45, Propositions 1.5.3 and 1.6.2, and Note 1.6]). Formally speaking, the norm $\|\cdot\|_{L}$ does not satisfy the Banach algebra law $\|f g\| \leq\|f\|\|g\|$. The best inequality assures that $\|f g\|_{L} \leq 2\|f\|_{L}\|g\|_{L}(f, g \in \operatorname{Lip}(E))$. We refer to the monograph [45] for the basic notions on Lipschitz algebras.

A. Jiménez-Vargas and M. Villegas-Vallecillos develop in [28] a detailed study on 2-local isometries on $\operatorname{Lip}(E)$. In [28, Theorem 2.1] they proved that for each bounded metric space $E$ such that $\operatorname{Iso}((\operatorname{Lip}(E)$, $\left.\left.\|\cdot\|_{L}\right)\right)$ is canonical, and each 2-local isometry $\Delta:\left(\operatorname{Lip}(E),\|\cdot\|_{L}\right) \rightarrow$ $\left(\operatorname{Lip}(E),\|\cdot\|_{L}\right)$, there exist a subset $E_{0}$ of $E$, a unimodular scalar $\tau$, and a bijective Lipschitz map $\varphi: E_{0} \rightarrow E$ such that

$$
\left.\Delta(f)\right|_{E_{0}} \equiv \tau(f \circ \varphi)
$$


for all $f \in \operatorname{Lip}(E)$, and the same statement holds when $\|\cdot\|_{L}$ is replaced by $\|\cdot\|_{s}$ (see Section 2 for the concrete definition of canonical surjective isometry). However, we do not have a complete control of $\Delta(f)$ outside $E_{0}$ to conclude that $\Delta$ is linear. If, under the above hypothesis, we additionally assume that $E$ is separable, then every 2-local isometry on $\left(\operatorname{Lip}(E),\|\cdot\|_{L}\right)$, or on $\left(\operatorname{Lip}(E),\|\cdot\|_{s}\right)$, is a surjective linear isometry (see [28, Theorem 3.3]). In this note we shall extend the study to weak-2-local isometries between $\operatorname{Lip}(E)$ algebras.

We are also interested in some other classes of function algebras. Suppose $K$ is a compact Hausdorff space. The norm closed subalgebras of $C(K)$ containing the constant functions and separating the points of $K$ (i.e., for every $t \neq s$ in $K$ there exists $f \in A$ such that $f(t) \neq f(s)$ ) are called uniform algebras. An abstract characterization of uniform algebras can be deduced from the Gelfand theory and the Gelfand-Beurling formula for the spectral radius; namely, if $A$ is a unital commutative complex Banach algebra such that $\left\|a^{2}\right\|=\|a\|^{2}$ for all $a$ in $A$, then there is a compact Hausdorff space $K$ such that $A$ is isomorphic as a Banach algebra to a uniform subalgebra of $C(K)$.

Useful examples of uniform algebras include certain spaces given by holomorphic properties. Suppose $K$ is a compact subset of $\mathbb{C}^{n}$, the algebra $A(K)$ of all complex valued continuous functions on $K$ which are holomorphic on the interior of $K$ is an example of uniform algebra. When $K=\mathbb{D}$ is the closed unit ball of $\mathbb{C}, A(\mathbb{D})$ is precisely the disc algebra. On the other hand, it is known that $\operatorname{Lip}(K)$ is norm dense in $\left(C(K),\|\cdot\|_{\infty}\right)[\mathbf{4 4}$, Exercise in p. 23], however there exist continuous functions which are not Lipschitz. Combining this fact with the StoneWeierstrass theorem, we deduce that $\operatorname{Lip}(K)$ is not, in general, a uniform algebra. The reader is referred to the monograph [20] for additional background on uniform algebras.

In [23] O. Hatori, T. Miura, H. Oka, and H. Takagi studied 2-local isometries and 2-local automorphisms between uniform algebras. These authors established some partial answers. As an application of the Kowalski-Słodkowski theorem (see Theorem 3.1), they first proved that for each uniform algebra $A$, every 2-local automorphism $T$ on $A$ is an isometrical isomorphism from $A$ onto $T(A)$. Furthermore, if the group of all automorphisms on $A$ is algebraically reflexive (i.e., if every local automorphism on $A$ is an automorphism), then every 2-local automorphism is an automorphism (see [23, Theorem 2.2]). They also showed the existence of non-surjective 2-local automorphisms on $C(K)$ spaces (see [23, Theorem 2.3]). For a compact subset $K \subseteq \mathbb{C} \operatorname{such}$ that $\operatorname{int}(K)$ 
is connected and $\overline{\operatorname{int}(K)}=K$, Hatori, Miura, Oka, and Takagi combined their results on local automorphism and local isometries on $A(K)$ with a previous contribution by F. Cabello Sánchez and L. Molnár (see [14]) to prove that every local isometry (respectively, every local automorphism) on $A(K)$ is a surjective isometry (respectively, is an automorphism) (see [23, Corollary 3.5]). Under certain restrictions on a set $K \subset \mathbb{C}$ or $K \subset \mathbb{C}^{2}$, the same authors established that every 2-local isometry (respectively, every 2-local automorphism) on $A(K)$ is a surjective linear isometry (respectively, an automorphism) [23, Theorems 3.6, 3.7, and 3.8]. In the same paper, these authors posed the following problems:

Problem 1.1 ([23, Problem 3.12]). Is every 2-local isometry on a uniform algebra linear?

Problem 1.2 ([23, Problem 3.13]). Is a 2-local isometry (respectively automorphism) on a uniform algebra a 3-local isometry (respectively automorphism)? In general, is an $n$-local isometry an $(n+1)$-local isometry?

We recall that given a natural $n$, a map $\Delta$ on a Banach algebra $B$ is called an $n$-local isometry (respectively, an $n$-local automorphism) if for every $n$-tuple $\left(a_{1}, a_{2}, \ldots, a_{n}\right)$ in $B$ there exists a surjective linear isometry (respectively, an automorphism) $S$ on $B$, depending on the elements in the tuple, such that $\Delta\left(a_{j}\right)=S\left(a_{j}\right)$ for every $1 \leq j \leq n$.

In this paper we give a complete positive answer to the above problems by proving that every weak-2-local isometry between uniform algebras is linear (see Theorem 3.10). This conclusion is actually stronger than what it was posed by Hatori, Miura, Oka, and Takagi. In order to prove this result we first establish appropriate spherical variants of the Gleason-Kahane-Żelazko and Kowalski-Słodkowski theorems (see Propositions 2.2 and 3.2). The spherical version of the Gleason-KahaneŻelazko theorem is employed in Section 2 to describe weak-local isometries on uniform algebras and on Lipschitz spaces.

Henceforth, let the symbol $\mathbb{T}$ stand for the unit sphere of $\mathbb{C}$. Throughout this note, the symbol $\sigma(a)$ will denote the spectrum of an element $a$ in a complex Banach algebra $A$. The key result in this note is a spherical variant of the Kowalski-Słodkowski theorem which assures that for an arbitrary complex unital Banach algebra $A$, a mapping $\Delta: A \rightarrow \mathbb{C}$ which is 1-homogeneous (i.e. $\Delta(\alpha x)=\alpha \Delta(x)$ for all $\alpha \in \mathbb{C}$ and $x \in A$ ) and satisfies that

$$
\Delta(x)-\Delta(y) \in \mathbb{T} \sigma(x-y), \text { for every } x, y \in A,
$$


must be linear. Furthermore, under these hypothesis, there exists $\lambda_{0}$ in $\mathbb{T}$ such that $\lambda_{0} \Delta$ is multiplicative (see Proposition 3.2).

We shall also apply the spherical variant of the Kowalski-Słodkowski theorem in the study of weak-2-local isometries between Lipschitz algebras. The concrete result reads as follows: Let $E$ and $F$ be metric spaces such that the set $\operatorname{Iso}\left(\left(\operatorname{Lip}(E),\|\cdot\|_{L}\right),\left(\operatorname{Lip}(F),\|\cdot\|_{L}\right)\right)$ is canonical. Then every weak-2-local $\operatorname{Iso}\left(\left(\operatorname{Lip}(E),\|\cdot\|_{L}\right),\left(\operatorname{Lip}(F),\|\cdot\|_{L}\right)\right)$-map $\Delta$ from $\operatorname{Lip}(E)$ to $\operatorname{Lip}(F)$ is a linear map. Furthermore, the same conclusion holds when the norm $\|\cdot\|_{L}$ is replaced by the norm $\|\cdot\|_{s}$ (see Theorem 3.5). Among the consequences of this result, we establish that for each compact metric space $K$ such that $\operatorname{Iso}\left(\operatorname{Lip}(K),\|\cdot\|_{s}\right)$ is canonical, every 2-local isometry $\Delta:\left(\operatorname{Lip}(K),\|\cdot\|_{s}\right) \rightarrow\left(\operatorname{Lip}(K),\|\cdot\|_{s}\right)$ is a surjective linear isometry (see Corollary 3.8).

\section{A spherical variant of the Gleason-Kahane-Żelazko theorem}

In this section we shall try to extend the study on 2-local isometries developed by Jiménez-Vargas and Villegas-Vallecillos in [28].

To understand the whole picture it is worth to recall the notions of local and weak-local maps. Following $[\mathbf{1 5}, \mathbf{1 2}]$, let $\mathcal{S}$ be a subset of the space $L(X, Y)$ of all linear maps between Banach spaces $X$ and $Y$. A linear mapping $\Delta: X \rightarrow Y$ is said to be a local $\mathcal{S}$-map (respectively, a weak-local $\mathcal{S}$-map) if for each $x \in X$ (respectively, if for each $x \in X$ and $\phi \in Y^{*}$ ), there exists $T_{x} \in \mathcal{S}$, depending on $x$ (respectively, there exists $T_{x, \phi} \in \mathcal{S}$, depending on $x$ and $\phi$ ), satisfying $\Delta(x)=T_{x}(x)$ (respectively, $\left.\phi \Delta(x)=\phi T_{x, \phi}(x)\right)$. When $\mathcal{S}$ is the set $\operatorname{Iso}(X, Y)$ of all surjective isometries from $X$ onto $Y$, weak-local Iso $(X, Y)$-maps are called weak local isometries. Local and weak-local maps have been intensively studied by a long list of authors (see, for example, $[\mathbf{7}, \mathbf{8}, \mathbf{1 1}, \mathbf{1 4}, \mathbf{1 5}, \mathbf{1 6}, \mathbf{2 6}, \mathbf{2 9}, \mathbf{3 1}, \mathbf{3 5}]$ and $[36])$.

It is known that every weak-local isometry $\Delta: X \rightarrow Y$ is contractive. Namely, for each $x \in X$ and $\phi \in Y^{*}$, there exists $T_{x, \phi} \in \operatorname{Iso}(X, Y)$, depending on $x$ and $\phi$, satisfying $\phi \Delta(x)=\phi T_{x, \phi}(x)$. Therefore, $|\phi \Delta(x)| \leq$ $\|\phi\|\left\|T_{x, \phi}(x)\right\|=\|\phi\|\|x\|$, for all $x \in X$ and $\phi \in Y^{*}$. It follows from the Hahn-Banach theorem that $\|\Delta(x)\| \leq\|x\|$ for all $x \in X$.

In their study on local isometries between Lipschitz algebras, A. Jiménez-Vargas, A. Morales Campoy, and M. Villegas-Vallecillos revealed the connection with the Gleason-Kahane-Żelazko theorem (see [26, p. 199]); a similar strategy was applied by F. Cabello Sánchez and L. Molnár in [14] for local isometries on a uniform algebra. Here, we shall try 
to determine the connection between 2-local isometries on complex-valued Lipschitz algebras and new subtle generalizations of the GleasonKahane-Żelazko and Kowalski-Słodkowski theorems. Let us recall the statement of these results. We briefly recall that, accordingly to standard references (see [6]), a complex Banach algebra is an associative algebra $A$ over the complex field which is also a Banach space satisfying

$$
\|x y\| \leq\|x\|\|y\|, \quad \forall x, y \in A .
$$

Theorem 2.1 (Gleason-Kahane-Żelazko theorem $[\mathbf{2 1}, \mathbf{3 2}, 46])$. Let $F: A \rightarrow \mathbb{C}$ be a non-zero continuous linear functional, where $A$ is a complex Banach algebra. Then the following statements are equivalent:

(a) $F(a) \in \sigma(a)$, for every $a \in A$.

(b) $F$ is multiplicative.

We begin with a technical spherical reformulation of the GleasonKahane-Żelazko theorem.

Proposition 2.2. Let $F: A \rightarrow \mathbb{C}$ be a continuous linear functional, where $A$ is a unital complex Banach algebra. Suppose that $F(a) \in \mathbb{T} \sigma(a)$, for every $a \in A$. Then the mapping $\overline{F(1)} F$ is multiplicative.

Proof: The arguments are very similar to those in $[\mathbf{3 2}, \mathbf{4 6}]$, we shall insert here a brief revision containing the required changes.

We fix $a$ in $A$, and define a mapping $\varphi: \mathbb{C} \rightarrow \mathbb{C}, \varphi(\lambda)=F\left(e^{\lambda a}\right)$. Since $F$ is linear and continuous, $\varphi(\lambda)=\sum_{n=0}^{\infty} \frac{F\left(a^{n}\right)}{n !} \lambda^{n}$ for all $\lambda \in \mathbb{C}$. Clearly, $\varphi$ is an entire function. By the assumptions $\varphi(\lambda) \in \mathbb{T} \sigma\left(e^{\lambda a}\right)$, and hence $\varphi(\lambda) \neq 0$ for every $\lambda \in \mathbb{C}$. It is known that every entire function which never vanishes admits a "logarithm", that is, there exists $\psi: \mathbb{C} \rightarrow \mathbb{C}$ entire such that $\varphi(\lambda)=e^{\psi(\lambda)}(\lambda \in \mathbb{C})$.

We also know that the inequality

$$
|\varphi(\lambda)| \leq \sum_{n=0}^{\infty} \frac{\left|F\left(a^{n}\right)\right|}{n !}|\lambda|^{n} \leq\|F\| e^{|\lambda|\|a\|}
$$

holds for every complex $\lambda$, and hence $\varphi$ has exponential type bounded by one. It follows from Hadamard's Factorization theorem (see [5, Theorem 2.7.1]) that $\psi(\lambda)=\alpha \lambda+\beta$ for suitable $\alpha, \beta \in \mathbb{C}$.

Therefore,

$$
\sum_{n=0}^{\infty} \frac{F\left(a^{n}\right)}{n !} \lambda^{n}=\varphi(\lambda)=e^{\alpha \lambda+\beta}=e^{\beta} \sum_{n=0}^{\infty} \frac{\alpha^{n}}{n !} \lambda^{n},
$$


for all $\lambda \in \mathbb{C}$. Consequently, $F\left(a^{n}\right)=e^{\beta} \alpha^{n}$ for all natural $n$, in particular $e^{-\beta} F(a)=\alpha$ and $e^{-\beta} F\left(a^{2}\right)=\alpha^{2}=\left(e^{-\beta} F(a)\right)^{2}$.

We have shown that the mapping $G(a):=e^{-\beta} F(a)$ is a linear functional with $G\left(a^{2}\right)=G(a)^{2}$ for every $a \in A$, that is, $G$ is a Jordan homomorphism (i.e., $G$ preserves products of the form $a \circ b=\frac{1}{2}(a b+b a)$ ). The arguments in [46] can be literally applied to conclude that $G$ is multiplicative (see also [6, Proposition 16.6]).

Another application of the hypothesis gives $e^{\beta}=\varphi(0)=F(1)=\overline{\lambda_{0}} \in$ $\mathbb{T}$, and hence $\overline{F(1)} F=G$ is multiplicative.

F. Cabello Sánchez and L. Molnár proved in [14, Theorem 5] that for each uniform algebra $A$, every local isometry $T$ on $A$ has the form

$$
T(f)=\tau \psi(f), \quad \forall f \in A,
$$

where $\tau$ is a unimodular element in $A$, and $\psi: A \rightarrow A$ is a unital algebra endomorphism. Actually, the result of Cabello Sánchez and Molnár only requires that for each $f \in A$, the element $T(f)$ lies in the norm closure of $\operatorname{Iso}(A)(f)=\{S(f): S \in \operatorname{Iso}(A)\}$. Furthermore, if $A=A(\mathbb{D})$ is the disc algebra, then every local isometry on $A(\mathbb{D})$ is a surjective linear isometry (see [14, Theorem 6]). The spherical version of the Gleason-KahaneŻelazko theorem established in Proposition 2.2 provides a powerful tool to extend the study by Cabello and Molnár to the setting of weak-local isometries between uniform algebras.

Theorem 2.3. Let $T: A \rightarrow B$ be a weak-local isometry between uniform algebras. Then there exists a unimodular element $u \in B$ and a unital algebra homomorphism $\psi: A \rightarrow B$ such that

$$
T(f)=u \psi(f), \quad \forall f \in A .
$$

Proof: Suppose $B$ is a norm-closed subalgebra of some $C(Q)$. Let us fix $s \in Q$, and consider the linear mapping $\delta_{s} \circ T: A \rightarrow \mathbb{C}$. The hypothesis combined with [17, Corollary 2.3.16] imply that, for each $f \in A$, there exist a unimodular element $u_{s, f} \in B$ and a unital algebra isomorphism $\psi_{s, f}: A \rightarrow B$ such that

$$
\delta_{s} \circ T(f)=T(f)(s)=u_{s, f}(s) \psi_{s, f}(f)(s) \in \mathbb{T} \sigma(f),
$$

because $u_{s, f}(s) \in \mathbb{T}$, and since $\psi_{s, f}$ a unital algebra isomorphism, we have $\psi_{s, f}(f)(s) \in \sigma\left(\psi_{s, f}(f)\right)=\sigma(f)$. 
We have already commented that weak-local isometries are contractive. Therefore $\delta_{s} \circ T$ is a continuous linear mapping. Furthermore, for the unit element $1 \in A$ there exist a unimodular element $u_{s, 1} \in B$ and a unital algebra isomorphism $\psi_{s, 1}: A \rightarrow B$ such that $\delta_{s} \circ T(1)=T(1)(s)=$ $u_{s, 1}(s) \psi_{s, 1}(1)(s)=u_{s, 1}(s) \in \mathbb{T}$. This shows that $\delta_{s} \circ T$ is surjective.

By Proposition 2.2 the mapping $\overline{T(1)(s)}\left(\delta_{s} \circ T\right): A \rightarrow \mathbb{C}$ is a homomorphism. We also know from the hypothesis and [17, Corollary 2.3.16] that $T(1)$ is a unimodular mapping and $\overline{T(1)}=T(1) \in B$. Since $s$ was arbitrarily chosen, we deduce that

$$
\overline{T(1)(s)}\left(\delta_{s} \circ T\right)(f g)=\overline{T(1)(s)}\left(\delta_{s} \circ T\right)(f) \overline{T(1)(s)}\left(\delta_{s} \circ T\right)(g),
$$

for all $f, g \in A$, equivalently $\psi=\overline{T(1)} T: A \rightarrow B$ is a homomorphism and $T=T(1) \psi$.

Henceforth, the symbol $S_{\mathbb{F}}$ will denote the unit sphere of $\mathbb{F}$.

Back to the setting of Lipschitz algebras, we recall that given a surjective isometry $\varphi: F \rightarrow E$ between two metric spaces, and an element $\tau \in S_{\mathbb{F}}$, the mapping

$$
T_{\tau, \varphi}: \operatorname{Lip}(E) \rightarrow \operatorname{Lip}(F), \quad T_{\tau, \varphi}(f)(s)=\tau f(\varphi(s)), \quad \forall f \in \operatorname{Lip}(E),
$$

is an element in $\operatorname{Iso}(\operatorname{Lip}(E), \operatorname{Lip}(F))$. Fortunately or not, there exist elements in $\operatorname{Iso}(\operatorname{Lip}(E), \operatorname{Lip}(F))$ which cannot be written as weighted composition operator via a surjective isometry $\varphi$ and $\tau \in S_{\mathbb{F}}$ as in the previous example (cf. $[44$, p. 242] or $[45, \S 2.6])$. The elements in Iso( $\operatorname{Lip}(E), \operatorname{Lip}(F))$ which can be written as weighted composition operators via a surjective isometry $\varphi$ and $\tau \in S_{\mathbb{F}}$ as above are called canonical. We shall say that $\operatorname{Iso}(\operatorname{Lip}(E), \operatorname{Lip}(F))$ is canonical if every element in this set is canonical.

Many attempts have been conducted to determine when the set

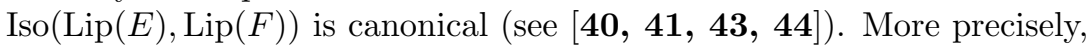
$\operatorname{Iso}\left(\operatorname{Lip}([0,1]),\|\cdot\|_{s}\right)$ is canonical (see $[\mathbf{4 0}]$ ) and, for a compact and connected metric space $K$ with diameter at most $1, \operatorname{Iso}\left(\operatorname{Lip}(K),\|\cdot\|_{L}\right)$ is canonical (cf. $[\mathbf{4 1}, \mathbf{4 3}]$ ). K. Jarosz and V. D. Pathak established in [25, Examples 8 and 3] that for every compact metric space $K$, $\operatorname{Iso}\left(\operatorname{Lip}(K),\|\cdot\|_{s}\right)$ is canonical, and under a certain separation property, $\operatorname{Iso}\left(\operatorname{Lip}(K),\|\cdot\|_{L}\right)$ is canonical too. It should be noted here that, as pointed out by $\mathrm{N}$. Weaver in [44, p. 243], there is a gap in the arguments applied in [25, Example 8]. The difficulties in the proof by Jarosz and Pathak mentioned in this paragraph have been completely solved in a recent paper by Hatori and Oi [24]. 
N. Weaver introduced subtle novelties in [44] by proving that if $E$ and $F$ are complete metric spaces of diameter $\leq 2$ and 1-connected then $\operatorname{Iso}\left(\left(\operatorname{Lip}(E),\|\cdot\|_{L}\right),\left(\operatorname{Lip}(F),\|\cdot\|_{L}\right)\right)$ is canonical (see $[\mathbf{4 4}$, Theorem D] or [45, Theorem 2.6.7]).

In the introduction of $[44]$ (see also $[45, \S 1.7]$ ), Weaver illustrated why it is worth to restrict our study to the class of complete metric spaces of diameter $\leq 2$, essentially because given a metric space $(E, d)$, we can consider the metric space $\left(F_{0}, d^{\prime}\right)$ whose underlying set is $E$ and whose distance is given by $d^{\prime}(x, y)=\min \{d(x, y), 2\}$, whose completion is denoted by $\left(F, d^{\prime}\right)$. Then $F$ is a complete metric space of diameter $\leq 2$ and $\operatorname{Lip}(E)$ and $\operatorname{Lip}(F)$ are isometrically isomorphic as Banach spaces.

Let us observe that while $\left(\operatorname{Lip}(E),\|\cdot\|_{s}\right)$ is a commutative and unital complex Banach algebra, the algebra $\left(\operatorname{Lip}(E),\|\cdot\|_{L}\right)$ only satisfies the weaker inequality

$$
\|f g\|_{L} \leq 2\|f\|_{L}\|g\|_{L}, \quad \forall f, g \in \operatorname{Lip}(E)
$$

(see $[45, \S 4.1])$.

In [26] A. Jiménez-Vargas, A. Morales Campoy, and M. VillegasVallecillos proved the following result:

Theorem 2.4 ([26, Theorem 2.3]). Let $E$ be a compact metric space. Then every local isometry on $\left(\operatorname{Lip}(E),\|\cdot\|_{s}\right)$ is a surjective isometry.

Next, thanks to the spherical version of the Gleason-Kahane-Żelazko theorem, we extend the study to weak-local isometries between Lipschitz spaces when they are indistinctly equipped with the norm $\|\cdot\|_{s}$ or with the norm $\|\cdot\|_{L}$.

Theorem 2.5. Let $E$ and $F$ be metric spaces. Then the following statements hold:

(a) Suppose that the set $\operatorname{Iso}\left(\left(\operatorname{Lip}(E),\|\cdot\|_{s}\right),\left(\operatorname{Lip}(F),\|\cdot\|_{s}\right)\right)$ is canonical. Then every weak-local isometry $T:\left(\operatorname{Lip}(E),\|\cdot\|_{s}\right) \rightarrow\left(\operatorname{Lip}(F),\|\cdot\|_{s}\right)$ can be written in the form

$$
T(f)=\tau \psi(f), \quad \forall f \in \operatorname{Lip}(E),
$$

where $\tau \in \operatorname{Lip}(F)$ is unimodular, and $\psi: \operatorname{Lip}(E) \rightarrow \operatorname{Lip}(F)$ is an algebra homomorphism.

(b) Suppose that the set $\operatorname{Iso}\left(\left(\operatorname{Lip}(E),\|\cdot\|_{L}\right),\left(\operatorname{Lip}(F),\|\cdot\|_{L}\right)\right)$ is canonical. Then every weak-local isometry $T:\left(\operatorname{Lip}(E),\|\cdot\|_{L}\right) \rightarrow(\operatorname{Lip}(F)$, $\left.\|\cdot\|_{L}\right)$ can be written in the form

$$
T(f)=\tau \psi(f), \quad \forall f \in \operatorname{Lip}(E),
$$

where $\tau \in \operatorname{Lip}(F)$ is unimodular, and $\psi: \operatorname{Lip}(E) \rightarrow \operatorname{Lip}(F)$ is an algebra homomorphism. 
Proof: We shall give a unified proof for both statements. To simplify the notation let $T:(\operatorname{Lip}(E),\|\cdot\|) \rightarrow(\operatorname{Lip}(F),\|\cdot\|)$ be a weak-local isometry, where $\|\cdot\|$ denotes $\|\cdot\|_{L}$ or $\|\cdot\|_{s}$. By the Hahn-Banach theorem every weak-local isometry is continuous and contractive.

By hypothesis, for each $s \in F$, there exist $\tau_{s, 1} \in \mathbb{T}$ and a surjective isometry $\varphi_{s, 1}: F \rightarrow E$ such that

$$
T(1)(s)=\delta_{s}(T(1))=\tau_{s, 1} 1\left(\varphi_{s, 1}(s)\right)=\tau_{s, 1} .
$$

Therefore $T(1)(s) \in \mathbb{T}$ for all $s \in F$. Similar arguments show that for each $f \in \operatorname{Lip}(E)$, there exists $\tau_{s, f} \in \mathbb{T}$ and a surjective isometry $\varphi_{s, f}: F \rightarrow E$ such that

$$
T(f)(s)=\delta_{s}(T(f))=\tau_{s, f} f\left(\varphi_{s, f}(s)\right) \in \mathbb{T} \sigma_{\left(\operatorname{Lip}(E),\|\cdot\|_{s}\right)}(f) .
$$

In case (a) we know that $\left(\operatorname{Lip}(E),\|\cdot\|_{s}\right)$ is a commutative unital complex Banach algebra, and $\delta_{s} \circ T: \operatorname{Lip}(E) \rightarrow \mathbb{C}$ is continuous. We can therefore apply Proposition 2.2 to conclude that

$$
\overline{T(1)(s)}\left(\delta_{s} \circ T\right):\left(\operatorname{Lip}(E),\|\cdot\|_{s}\right) \rightarrow \mathbb{C}
$$

is a homomorphism for all $s \in F$. Consequently, the operator $\psi=$ $\overline{T(1)} T: \operatorname{Lip}(E) \rightarrow \operatorname{Lip}(F)$ is a homomorphism and $T(f)=T(1) \psi(f)$, for all $f \in \operatorname{Lip}(E)$.

In case (b), as we have commented above, $\left(\operatorname{Lip}(E),\|\cdot\|_{L}\right)$ is not strictly a Banach algebra (see comments before Theorem 2.4). However, the algebras underlying $\left(\operatorname{Lip}(E),\|\cdot\|_{s}\right)$ and $\left(\operatorname{Lip}(E),\|\cdot\|_{L}\right)$ are both the same. Furthermore, for each $s \in F$ the mapping $\delta_{s} \circ T:\left(\operatorname{Lip}(E),\|\cdot\|_{L}\right) \rightarrow \mathbb{C}$ can be also considered as a continuous functional on $\left(\operatorname{Lip}(E),\|\cdot\|_{s}\right)$ satisfying the hypothesis of Proposition 2.2; we can therefore apply case (a) to conclude the proof.

We observe that statement (b) in the above Theorem 2.5 is a new result even in the setting of local isometries. When the group of surjective isometries is not canonical the spherical Gleason-Kahane-Żelazko theorem can not be applied. For example, let $E=\{p, q\}$ be the compact metric space consisting of two elements with $d(p, q)=1$. We can identify $\operatorname{Lip}(E)$ with $\mathbb{C}^{2}$ with norm $\|(a, b)\|_{L}=\max \{|a|,|b|,|a-b|\}$. The mapping $T: \operatorname{Lip}(E) \rightarrow \operatorname{Lip}(E)$ defined by $T(a, b)=(a, a-b)$ is a surjective linear isometry which is not canonical (see $[\mathbf{4 4}]$ ). Furthermore, the functional $\delta_{q} \circ T: \operatorname{Lip}(E) \rightarrow \mathbb{C},(a, b) \mapsto a-b$ does not satisfy that $\delta_{q} \circ T(f) \in \mathbb{T} f(E)=\mathbb{T} \sigma(f)$ for all $f \in \operatorname{Lip}(E)$.

Remark 2.6. If in Theorem 2.5 the hypothesis

$$
\operatorname{Iso}\left(\left(\operatorname{Lip}(E),\|\cdot\|_{s}\right),\left(\operatorname{Lip}(F),\|\cdot\|_{s}\right)\right) \text { being canonical }
$$


(respectively, $\operatorname{Iso}\left(\left(\operatorname{Lip}(E),\|\cdot\|_{L}\right),\left(\operatorname{Lip}(F),\|\cdot\|_{L}\right)\right)$ being canonical) is replaced by the weaker assumption that every element $T$ in the set $\operatorname{Iso}\left(\left(\operatorname{Lip}(E),\|\cdot\|_{s}\right),\left(\operatorname{Lip}(F),\|\cdot\|_{s}\right)\right)$ (respectively, in the set $\operatorname{Iso}((\operatorname{Lip}(E)$, $\left.\left.\left.\|\cdot\|_{L}\right),\left(\operatorname{Lip}(F),\|\cdot\|_{L}\right)\right)\right)$ is of the form

$$
T(f)(s)=\tau(s) f(\varphi(s)), \quad \forall f \in \operatorname{Lip}(E), \forall s \in F,
$$

where $\tau$ is a unimodular function $\operatorname{in} \operatorname{Lip}(F)$ and $\varphi: F \rightarrow E$ is a surjective isometry, then the conclusion of the quoted Theorem 2.5 remains true.

\section{A spherical variant of the Kowalski-Słodkowski theorem}

The Kowalski-Słodkowski theorem is less widely known than the Gleason-Kahane-Żelazko theorem, however it shows that at the cost of requiring a 2-local behavior, the linearity assumption in the GleasonKahane-Żelazko theorem can be relaxed. Actually, the KowalskiSłodkowski theorem was the motivation to consider 2-local automorphisms (see [42]). The concrete result reads as follows.

Theorem 3.1 (Kowalski-Słodkowski theorem [33]). Let $A$ be a complex Banach algebra, and let $\Delta: A \rightarrow \mathbb{C}$ be a mapping satisfying $\Delta(0)=0$ and

$$
\Delta(x)-\Delta(y) \in \sigma(x-y)
$$

for every $x, y \in A$. Then $\Delta$ is linear and multiplicative.

As in the case of the Gleason-Kahane-Żelazko theorem, we shall establish a spherical variant of the Kowalski-Słodkowski theorem. We shall say that a mapping $\Delta: X \rightarrow Y$ between complex Banach spaces is 1-homogeneous or simply homogeneous if $\Delta(\lambda x)=\lambda \Delta(x)$, for every $x \in X, \lambda \in \mathbb{C}$.

Proposition 3.2. Let $A$ be a unital complex Banach algebra, and let $\Delta: A \rightarrow \mathbb{C}$ be a mapping satisfying the following properties:

(a) $\Delta$ is 1-homogeneous.

(b) $\Delta(x)-\Delta(y) \in \mathbb{T} \sigma(x-y)$, for every $x, y \in A$.

Then $\Delta$ is linear, and there exists $\lambda_{0} \in \mathbb{T}$ such that $\lambda_{0} \Delta$ is multiplicative.

Let $\mathcal{S}$ be a subset of the space $L(X, Y)$ of all linear maps between two complex Banach spaces. Suppose that $\Delta: X \rightarrow Y$ is a weak-2-local $\mathcal{S}$ map in the sense of [12] (i.e. for every $x, y \in X$ and $\phi \in Y^{*}$, there exists $T_{x, y, \phi} \in \mathcal{S}$, depending on $x, y$, and $\phi$ such that $\phi \Delta(x)=\phi T_{x, y, \phi}(x)$ and $\left.\phi \Delta(y)=\phi T_{x, y, \phi}(y)\right)$. It follows from [12, Lemma 2.1] that every weak2-local Iso $(X, Y)$ is 1-homogeneous. So, the hypothesis in the above proposition are fully motivated in the study of weak-2-local isometries. 
The arguments here are somehow inspired by the original ideas due to Kowalski and Słodkowski. Before dealing with the proof of the above proposition we establish a variant of [33, Lemma 2.1]. Let us first make an observation. Given a compact Hausdorff space $K$ and $t \in K$, the mapping $\bar{\delta}_{t}: C(K) \rightarrow \mathbb{C}, f \mapsto \bar{\delta}_{t}(f)=\overline{f(t)}$ is conjugate linear, and satisfies the property that $\bar{\delta}_{t}(f) \in \mathbb{T} \sigma(f)$, for each $f \in C(K)$. So, the just quoted property is not enough to guarantee that a real linear functional is complex linear.

Lemma 3.3. Let $A$ be a unital complex Banach algebra, and let $F: A \rightarrow$ $\mathbb{C}$ be a bounded real linear functional satisfying the following property:

$$
F(x) \in \mathbb{T} \sigma(x), \text { for every } x \in A .
$$

Then $F$ is complex linear or conjugate linear.

Proof: In a first step we assume that $F$ is unital, that is $F(1)=1$. Fix an element $x \in A$. By hypothesis, we have

$$
e^{i t} F\left(e^{-i t} x\right) \in e^{i t} \mathbb{T} \sigma\left(e^{-i t} x\right)=\mathbb{T} \sigma(x)
$$

for each real $t$. We can write

$$
e^{i t} F\left(e^{-i t} x\right)=\frac{F(x)-i F(i x)}{2}+e^{2 i t} \frac{F(x)+i F(i x)}{2},
$$

which shows that $e^{i t} F\left(e^{-i t} x\right)$ is precisely the circle $S\left(\frac{F(x)-i F(i x)}{2}, \rho_{x}\right)$ of center $\frac{F(x)-i F(i x)}{2}$ and radius $\rho_{x}=\left|\frac{F(x)+i F(i x)}{2}\right|$, and consequently this circle is entirely contained in $\mathbb{T} \sigma(x)$. It is not hard to check that the functionals

$$
\begin{aligned}
& F_{1}(x):=\Re \mathrm{e} F(x)+i \Im \mathrm{m}(-i F(i x))=\Re \mathrm{e} F(x)-i \Re \mathrm{e} F(i x), \\
& F_{2}(x):=\Re \mathrm{e}(-i F(i x))+i \Im \mathrm{m} F(x)=\Im \mathrm{m} F(i x)+i \Im \mathrm{m} F(x)
\end{aligned}
$$

are continuous and satisfy the following properties:

(1) $F_{j}(i x)=i F_{j}(x)$ for all $j=1,2$ and all $x \in A$, and hence $F_{1}$ and $F_{2}$ are complex linear.

(2) $F_{j}(x) \in S\left(\frac{F(x)-i F(i x)}{2}, \rho_{x}\right) \subseteq \mathbb{T} \sigma(x)$, for every $x \in A, j=1,2$.

(3) $\Re \mathrm{e}(F(x))=\Re \mathrm{e}\left(F_{1}(x)\right)$ and $\Im \mathrm{m}(F(x))=\Im \mathrm{m}\left(F_{2}(x)\right)$ for all $x \in A$.

Property (2) implies that we can apply Proposition 2.2 to conclude that $G_{1}=\overline{F_{1}(1)} F_{1}$ and $G_{2}=\overline{F_{2}(1)} F_{2}$ are multiplicative functionals.

It also follows from $(2)$ that $F_{1}(1) \in \mathbb{T}$. Since $F(1)=1$ we have

$$
\mathbb{T} \ni F_{1}(1)=\Re \mathrm{e} F(1)-i \Re \mathrm{e} F(i 1)=1-i \Re \mathrm{e} F(i 1),
$$

which assures that $\Re \mathrm{e} F(i 1)=0$ and hence $F_{1}(1)=1$. This shows that $F_{1}=G_{1}$ is multiplicative. 
Similarly, from (2) and $F(1)=1$ we get

$$
\mathbb{T} \ni F_{2}(1)=\Im \mathrm{m} F(i 1)+i \Im \mathrm{m} F(1)=\Im \mathrm{m} F(i 1),
$$

witnessing that $F_{2}(1)=\Im \mathrm{m} F(i 1)= \pm 1$.

We claim that

$$
F_{2}=F_{2}(1) F_{1} \text {. }
$$

Indeed, if $\operatorname{ker}\left(F_{2}\right) \subseteq \operatorname{ker}\left(F_{1}\right)$, then for each $x \in A$ we have $x-F_{2}(1) F_{2}(x) 1 \in$ $\operatorname{ker}\left(F_{2}\right) \subseteq \operatorname{ker}\left(F_{1}\right)$, and thus $F_{1}(x)=F_{2}(1) F_{2}(x)$. If $F_{2}(1)=1$, then $F_{2}=F_{1}$, and hence property (3) above implies that $F=F_{1}$ is $\mathbb{C}$-linear. If $F_{2}(1)=-1$, then $F_{2}=-F_{1}$, and then property (3) implies that $F=\overline{F_{1}}$ is conjugate linear.

If $\operatorname{ker}\left(F_{2}\right) \nsubseteq \operatorname{ker}\left(F_{1}\right)$, then we can thus find an element $x_{0}$ in $A$ such that $F_{1}\left(x_{0}\right)=1$ and $F_{2}\left(x_{0}\right)=0$.

Let $h: \mathbb{C} \rightarrow \mathbb{C}$ denote the entire function given by $h(\lambda)=e^{i \frac{\pi}{2} \lambda}-1$. Having in mind that $F_{1}$ and $G_{2}$ are multiplicative, we deduce from the spectral mapping theorem and (3) that

$$
\begin{aligned}
F\left(e^{i \frac{\pi}{2} x_{0}}\right)-1 & =F\left(e^{i \frac{\pi}{2} x_{0}}-1\right)=F\left(h\left(x_{0}\right)\right) \\
& =\Re \mathrm{e}\left(F\left(h\left(x_{0}\right)\right)\right)+i \Im \mathrm{m}\left(F\left(h\left(x_{0}\right)\right)\right) \\
& =\Re \mathrm{e}\left(F_{1}\left(h\left(x_{0}\right)\right)\right)+i \Im \mathrm{m}\left(F_{2}\left(h\left(x_{0}\right)\right)\right) \\
& =\Re \mathrm{e}\left(F_{1}\left(h\left(x_{0}\right)\right)\right)+i \Im \mathrm{m}\left(F_{2}(1) G_{2}\left(h\left(x_{0}\right)\right)\right) \\
& =\Re \mathrm{e}\left(h\left(F_{1}\left(x_{0}\right)\right)\right)+i \Im \mathrm{m}\left(F_{2}(1) h\left(G_{2}\left(x_{0}\right)\right)\right) \\
& =\Re \mathrm{e}(h(1))+i \Im \mathrm{m}\left(F_{2}(1) h(0)\right)=-1,
\end{aligned}
$$

and consequently $F\left(e^{i \frac{\pi}{2} x_{0}}\right)=0$. On the other hand, it follows from our hypothesis and the spectral mapping theorem that

$$
0=F\left(e^{i \frac{\pi}{2} x_{0}}\right) \in \mathbb{T} \sigma\left(e^{i \frac{\pi}{2} x_{0}}\right)=\mathbb{T} e^{i \frac{\pi}{2} \sigma\left(x_{0}\right)} \subseteq \mathbb{T} e^{\mathbb{C}},
$$

which is impossible.

In the general case, the hypothesis imply that $F(1) \in \mathbb{T} \sigma(1)=\mathbb{T}$. The mapping $G=\overline{F(1)} F: A \rightarrow \mathbb{C}$ is real linear, unital, and satisfies

$$
G(x)=\overline{F(1)} F(x) \in \overline{F(1)} \mathbb{T} \sigma(x)=\mathbb{T} \sigma(x) .
$$

It follows from the previous case that $G$ (and hence $F$ ) is complex linear or conjugate linear.

Lemma 3.3 provides a tool to replace [33, Lemma 2.1] in the KowalskiSłodkowski theorem.

Before dealing with the proof of Proposition 3.2 we introduce some terminology. Let $\mathcal{Q}=\prod_{j=1}^{\infty}\left[-2^{-j}, 2^{j}\right] \subseteq \ell_{1}$ denote the Hilbert cube equipped 
with the metric given by $\ell_{1}$. On each interval $\left[-2^{-j}, 2^{j}\right]$ we consider the normalized Lebesgue measure. Let $\mu$ denote the natural product measure on $\mathcal{Q}$. Following $[\mathbf{3 3}, \mathbf{3 7}]$, we shall say that a subset $Z$ of a separable Banach space $X$ is a zero set if for every affine continuous mapping $j: \mathcal{Q} \rightarrow X$ with linearly dense image we have $\mu\left(j^{-1}(Z)\right)=0$.

Following the notation in $[\mathbf{3 7}, \S 4]$, we shall say that a Lipschitz mapping $F$ from the Hilbert cube $\mathcal{Q}$ into a locally convex space $X$ is real differentiable at the point $p \in \mathcal{Q}$ if for every $a \in \mathcal{Q}$ the derivative

$$
F_{a}^{\prime}(p)=\lim _{\mathbb{R} \ni t \rightarrow 0} \frac{F(p+t a)-F(p)}{t}
$$

exists and the mapping $(D F)_{p}: \widetilde{\mathcal{Q}} \rightarrow X,(D F)_{p}(a)=F_{a}^{\prime}(p)$ is real linear, where $\widetilde{\mathcal{Q}}$ denotes the linear span of $\mathcal{Q}$ in $\ell_{1}$. The mapping $(D F)_{p}$ is called the real differential of $F$ at $p$. It is known that if the real differential $(D F)_{p}$ exists for some $p$ in $\mathcal{Q}$, then it is continuous. Similarly a Lipschitz mapping $F: X \rightarrow \mathbb{C}$ admits a real differential at a point $x$ in $X$ if for every $a \in X$ the derivative $F_{a}^{\prime}(x)=\lim _{\mathbb{R} \ni t \rightarrow 0} \frac{F(x+t a)-F(x)}{t}$ exists and the mapping $(D F)_{x}: X \rightarrow \mathbb{C},(D F)_{x}(a)=F_{a}^{\prime}(x)$ is real linear and continuous.

The next result has been borrowed from [33], where it is stated without an explicit proof, and it is attributed to P. Mankiewicz [37].

Theorem 3.4 ([33, Theorem 2.3] and [37, Theorem 4.4 and proof of Theorem 4.5]). If $F: X \rightarrow \mathbb{C}$ is a Lipschitz mapping defined on a separable Fréchet space, then $F$ admits real differentials except for some zero set.

The result and a brief comment for its proof have been included here to avoid misleadings. All the arguments are explicitly in [37, Theorem 4.4 and proof of Theorem 4.5]. Clearly, $\mathbb{C}$ is a Gelfand-Fréchet space in the terminology of the just quoted paper (see [37, Theorem 2.5]). Let $\left\{a_{n}: n \in \mathbb{N}\right\}$ be an arbitrary bounded, linearly independent and linearly dense subset of $X$. Given $\varepsilon>0$, we set $\mathcal{Q}_{\varepsilon}:=(1+\varepsilon) \mathcal{Q}$, and we define a mapping $F_{0}: \mathcal{Q}_{\varepsilon} \rightarrow X$ given by

$$
F_{0}(p):=\sum_{k=1}^{\infty} \alpha_{k} a_{k}, \text { where } p=\left(\alpha_{n}\right) \in \mathcal{Q}_{\varepsilon} .
$$

As observed by Mankiewicz, the mapping $F_{0}$ satisfies the first order Lipschitz condition, that is, for every continuous pseudo norm $Q(\cdot)$ on $X$ there exists a continuous pseudo norm $P(\cdot)$ on $\ell_{1}$ such that for every $p_{1}, p_{2} \in \mathcal{Q}_{\varepsilon}$ we have

$$
Q\left(F_{0}\left(p_{1}\right)-F_{0}\left(p_{2}\right)\right) \leq P\left(p_{1}-p_{2}\right) .
$$


Furthermore, the composition $F \circ F_{0}: \mathcal{Q}_{\varepsilon} \rightarrow \mathbb{C}$ is a Lipschitz mapping from $\mathcal{Q}_{\varepsilon}$ into $\mathbb{C}$. Theorem 4.4 in $[\mathbf{3 7}]$ assures that $F \circ F_{0}$ admits real differentials except for $\mu$-almost all $p$ in $\mathcal{Q}$, that is, $\left(D\left(F \circ F_{0}\right)\right)_{p}$ exists for all $p \in \mathcal{Q} \backslash Z_{0}$, where $Z_{0} \subset \mathcal{Q}_{\varepsilon}$ with $\mu\left(Z_{0}\right)=0$. It is explicitly shown in $[37$, pp. 25 and 26] that $F$ admits a real differential at every point in $F_{0}\left(\mathcal{Q} \backslash Z_{0}\right)$, that is, the real differential $(D F)_{F_{0}(p)}: X \rightarrow \mathbb{C}$ exists and it is a continuous real linear mapping for every $p_{0} \in \mathcal{Q} \backslash Z_{0}$.

Proof of Proposition 3.2: Since $\Delta$ is homogeneous, we can easily see that $\Delta(0)=0$.

As in the proof of [33, Theorem 1.2], we shall assume first that $A$ is separable.

Under the hypothesis of our proposition, given $x, y$ in $A$, we have $\Delta(x)-\Delta(y) \in \mathbb{T} \sigma(x-y)$ and hence

$$
|\Delta(x)-\Delta(y)| \leq\|x-y\|,
$$

which guarantees that $\Delta$ is a Lipschitz map. Theorem 3.4 (see also $[\mathbf{3 3}$, Theorem 2.3]) assures that $\Delta$ admits real differentials except for some zero set.

Suppose that $\Delta$ admits a real differential at a point $a$. The differential $(D \Delta)_{a}: A \rightarrow \mathbb{C}$ is a real linear mapping defined by

$$
(D \Delta)_{a}(x)=\lim _{r \rightarrow 0} \frac{\Delta(a+r x)-\Delta(a)}{r} .
$$

By assumptions, $\frac{\Delta(a+r x)-\Delta(a)}{r} \in \mathbb{T} \frac{\sigma(r x)}{r}=\mathbb{T} \sigma(x)$, and thus

$$
(D \Delta)_{a}(x) \in \mathbb{T} \sigma(x) .
$$

Lemma 3.3 proves that $(D \Delta)_{a}$ is complex linear or conjugate linear.

Let $a$ be an element in $A$ such that $\Delta$ admits a real differential at $a$ and $\Delta(a) \neq 0$. In this case, by the homogeneity of $\Delta$ we have

$$
\begin{aligned}
(D \Delta)_{a}(i a) & =\lim _{r \rightarrow 0} \frac{\Delta(a+i r a)-\Delta(a)}{r} \\
& =\lim _{r \rightarrow 0} \frac{(1+i r) \Delta(a)-\Delta(a)}{r}=i \Delta(a),
\end{aligned}
$$

and

$$
(D \Delta)_{a}(a)=\lim _{r \rightarrow 0} \frac{\Delta(a+r a)-\Delta(a)}{r}=\Delta(a) \neq 0,
$$

and consequently $(D \Delta)_{a}$ is complex linear. We have proven:

$$
\Delta(a) \neq 0 \text { and } \exists(D \Delta)_{a} \Rightarrow(D \Delta)_{a} \text { is complex linear. }
$$

Suppose now that $\Delta(a)=0$ and $\Delta$ admits a real differential at $a$. We shall prove that $(D \Delta)_{a}$ is complex linear. 
We first show that $(D \Delta)_{a}(1) \neq 0$. Since $\Delta$ is homogeneous and $\Delta(a)=0$, we also have $\Delta(\alpha a)=0$ for every $\alpha \in \mathbb{C}$. Indeed, by assumptions, given $r \in \mathbb{R} \backslash\{0\}$, the element

$$
\Delta\left(\frac{1}{r} a+1\right)=\Delta\left(\frac{1}{r} a+1\right)-\Delta\left(\frac{1}{r} a\right) \in \mathbb{T} \sigma(1)=\mathbb{T},
$$

and thus

$$
\begin{aligned}
(D \Delta)_{a}(1) & =\lim _{r \rightarrow 0} \frac{\Delta(a+r 1)-\Delta(a)}{r}=\lim _{r \rightarrow 0} \frac{\Delta(a+r 1)}{r} \\
& =\lim _{r \rightarrow 0} \Delta\left(\frac{1}{r} a+1\right) \in \mathbb{T},
\end{aligned}
$$

which proves the desired statement.

Let $U:=\{c \in A: \Delta(c) \neq 0\}$. Since $\Delta$ is a Lipschitz function and the set $U$ is open, it follows from $(3.2)$ that $(D \Delta)_{c}$ is complex linear for every point $c \in U$ such that $\Delta$ admits a real differential at $c$. Applying [33, Lemma 2.4] we conclude that $\Delta$ is holomorphic on $U$, more precisely, for each $c \in U$ and $b \in A$ there exists $\rho=\rho(c, b)>0$, depending on $c$ and $b$, such that $c+\lambda b \in U$, for every $|\lambda|<\rho$, and $f_{c, b}(\lambda)=\Delta(c+\lambda b)$ is holomorphic on $\{\lambda \in \mathbb{C}:|\lambda|<\rho\}$.

Having in mind that $\Delta(a)=0$, it follows from the hypothesis that

$$
\Delta(a+\alpha 1)=\Delta(a+\alpha 1)-\Delta(a) \in \mathbb{T} \sigma(\alpha 1)=\alpha \mathbb{T},
$$

and thus $\Delta(a+\alpha 1) \neq 0$, for all $\alpha \in \mathbb{C} \backslash\{0\}$, equivalently, $a+\alpha 1 \in U$, for all $\alpha \in \mathbb{C} \backslash\{0\}$.

We consider the continuous function $f: \mathbb{C} \rightarrow \mathbb{C}, f(\lambda)=\Delta(a+\lambda 1)$. We shall prove that $f$ is holomorphic in $\mathbb{C} \backslash\{0\}$. Namely, fix $\lambda_{0} \in \mathbb{C} \backslash\{0\}$. Since $c=a+\lambda_{0} 1 \in U$, it follows from the above paragraph with $b=1$ that there exists $\rho>0$ such that $c+\lambda b \in U$, for every $|\lambda|<\rho$, and $f_{c, b}(\lambda)=\Delta(c+\lambda b)=\Delta\left(a+\lambda_{0} 1+\lambda 1\right)=f\left(\lambda_{0}+\lambda\right)$ is holomorphic on $\{\lambda \in \mathbb{C}:|\lambda|<\rho\}$, witnessing that $f$ is holomorphic at $\lambda_{0}$.

Since $f$ is continuous on $\mathbb{C}$ and holomorphic in $\mathbb{C} \backslash\{0\}$ it must be an entire function. In particular there exists $f^{\prime}(0)=\lim _{h \in \mathbb{C}, h \rightarrow 0} \frac{f(h)-f(0)}{h}$, and

$$
\begin{aligned}
(D \Delta)_{a}(1) & =\lim _{r \rightarrow 0} \frac{\Delta(a+r 1)-\Delta(a)}{r}=\lim _{r \in \mathbb{R}, r \rightarrow 0} \frac{f(r)-f(0)}{r}=f^{\prime}(0) \\
& =\lim _{r \in \mathbb{R}, r \rightarrow 0} \frac{f(i r)-f(0)}{i r}=\lim _{r \rightarrow 0} \frac{\Delta(a+i r 1)-\Delta(a)}{i r}=\frac{1}{i}(D \Delta)_{a}(i 1) .
\end{aligned}
$$

Since $(D \Delta)_{a}(1) \neq 0,(D \Delta)_{a}(i 1)=i(D \Delta)_{a}(1)$, and $(D \Delta)_{a}$ must be complex linear or conjugate linear, we conclude that $(D \Delta)_{a}$ is complex linear. 
We have shown that $(D \Delta)_{a}$ is complex linear for all point $a \in A$ at which $\Delta$ admits a real differential.

We can therefore apply [33, Lemma 2.4] (with $U=A$ ) to conclude that for each $a, b \in A$ the mapping $\varphi: \mathbb{C} \rightarrow \mathbb{C}, \varphi(\lambda):=\Delta(\lambda a+b)$ is entire with $\varphi(\lambda)-\varphi(\mu) \in \mathbb{T} \sigma((\lambda-\mu) a)$, and thus $|\varphi(\lambda)-\varphi(\mu)| \leq$ $\|a\||\lambda-\mu|$. The mapping $g: \mathbb{C} \rightarrow \mathbb{C}, g(\lambda)=\varphi(\lambda)-\varphi(0)$ is entire and satisfies $|g(\lambda)| \leq\|a\||\lambda|$ for every $\lambda \in \mathbb{C}$. It follows from the so-called generalized Liouville's theorem that $g$ is a polynomial of degree at most 1 (compare [5, Theorem 1.3.4 and its proof]). Therefore $\varphi$ must be affine, and consequently,

$$
\Delta(\lambda a+b)=\varphi(\lambda)=\lambda(\Delta(a+b)-\Delta(b))+\Delta(b),
$$

for every $a, b \in A, \lambda \in \mathbb{C}$. In the case $b=0$ we get $\Delta(\lambda a)=\lambda \Delta(a)$, and given $c, d \in A$, by replacing in (3.3) $a, b$, and $\lambda$ with $\frac{c-d}{2}, d$, and 2 , respectively, we have

$$
2 \Delta\left(\frac{c+d}{2}\right)=\Delta(c)+\Delta(d) .
$$

So, $\Delta$ is linear and the rest follows from Proposition 2.2.

When $A$ is not separable, we can restrict $\Delta$ to the subalgebra generated by any two elements which is always separable, and then the conclusion follows from the arguments above.

Finally, since $\Delta: A \rightarrow \mathbb{C}$ is linear, $\Delta(0)=0$ and $\Delta(a) \in \mathbb{T} \sigma(a)$ for every $a$ in $A$, the final statement is a consequence of Proposition 2.2.

We can now deal with 2-local isometries between $\operatorname{Lip}(E)$ spaces.

Theorem 3.5. Let $E$ and $F$ be metric spaces, and let us assume that the set $\operatorname{Iso}\left(\left(\operatorname{Lip}(E),\|\cdot\|_{s}\right),\left(\operatorname{Lip}(F),\|\cdot\|_{s}\right)\right)$ is canonical. Then every weak-2local $\operatorname{Iso}\left(\left(\operatorname{Lip}(E),\|\cdot\|_{s}\right),\left(\operatorname{Lip}(F),\|\cdot\|_{s}\right)\right)$-map $\Delta$ from $\operatorname{Lip}(E)$ to $\operatorname{Lip}(F)$ is a linear map. Furthermore, the same conclusion holds when the norm $\|\cdot\|_{s}$ is replaced by the norm $\|\cdot\|_{L}$.

Proof: Let $\Delta: \operatorname{Lip}(E) \rightarrow \operatorname{Lip}(F)$ be a weak-2-local isometry with respect to the norm $\|\cdot\|_{s}$. It is known that $\Delta$ is 1-homogeneous (i.e., $\Delta(\alpha f)=$ $\alpha \Delta(f)$, for all $\alpha \in \mathbb{C}$ and all $f \in \operatorname{Lip}(E)$ ), and $\Delta(0)=0$ (compare [12, Lemma 2.1]). We fix now an element $s \in F$, and we consider the mapping $\Delta_{s}=\delta_{s} \circ \Delta: \operatorname{Lip}(E) \rightarrow \mathbb{C}$. Since, by hypothesis, given $f, g \in \operatorname{Lip}(E)$, there exist $\tau_{f, g, s} \in \mathbb{T}$ and a surjective isometry $\varphi_{f, g, s}: F \rightarrow E$ such that

$$
\delta_{s} \Delta(f)=\delta_{s}\left(\tau_{f, g, s} f \circ \varphi_{f, g, s}\right) \text {, and } \delta_{s} \Delta(g)=\delta_{s}\left(\tau_{f, g, s} g \circ \varphi_{f, g, s}\right),
$$

and then

$$
\Delta_{s}(f)-\Delta_{s}(g)=\tau_{f, g, s}\left(f\left(\varphi_{f, g, s}(s)\right)-g\left(\varphi_{f, g, s}(s)\right)\right) \in \mathbb{T} \sigma(f-g) .
$$


Since $\left(\operatorname{Lip}(E),\|\cdot\|_{s}\right)$ is a unital complex Banach algebra, we are thus in conditions to apply Proposition 3.2 to conclude that $\Delta_{s}$ is a linear map. The linearity of $\Delta$ follows from the arbitrariness of $s$.

For the last statement, the space $\left(\operatorname{Lip}(E),\|\cdot\|_{L}\right)$ is not formally a complex Banach algebra. However, by the arguments given above, for each weak-2-local isometry $\Delta:\left(\operatorname{Lip}(E),\|\cdot\|_{L}\right) \rightarrow\left(\operatorname{Lip}(F),\|\cdot\|_{L}\right)$ and each $s \in F$, we have

$$
\delta_{s} \circ \Delta(f)-\delta_{s} \circ \Delta(g) \in \mathbb{T}(f-g)(E)=\mathbb{T} \sigma_{\left(\operatorname{Lip}(E),\|\cdot\|_{s}\right)}(f-g),
$$

for all $f, g \in \operatorname{Lip}(E)$. Since the spaces and algebras underlying $(\operatorname{Lip}(E)$, $\left.\|\cdot\|_{L}\right)$ and $\left(\operatorname{Lip}(E),\|\cdot\|_{s}\right)$ coincide, we apply the conclusion in the first paragraph to the proof.

Remark 3.6. If in Theorem 3.5 the hypothesis

$$
\operatorname{Iso}((\operatorname{Lip}(E),\|\cdot\|),(\operatorname{Lip}(F),\|\cdot\|)) \text { being canonical }
$$

(where $\|\cdot\|$ stands for $\|\cdot\|_{s}$ or for $\|\cdot\|_{L}$ ), is replaced by the weaker assumption that every element in $\operatorname{Iso}((\operatorname{Lip}(E),\|\cdot\|),(\operatorname{Lip}(F),\|\cdot\|))$ is of the form

$$
T(f)(s)=\tau(s) f(\varphi(s)), \quad \forall f \in \operatorname{Lip}(E), \forall s \in F,
$$

where $\tau$ is a unimodular function $\operatorname{in} \operatorname{Lip}(F)$ and $\varphi: F \rightarrow E$ is a surjective isometry, then the conclusion of Theorem 3.5 remains valid.

It should be noted here that, under the hypothesis of the previous theorem (i.e., the set $\operatorname{Iso}\left(\left(\operatorname{Lip}(E),\|\cdot\|_{L}\right),\left(\operatorname{Lip}(F),\|\cdot\|_{L}\right)\right)$ is canonical), we can also prove a variant of $[\mathbf{2 8}$, Theorem 2.1] in the setting of 2-local isometries from $\operatorname{Lip}(E)$ to $\operatorname{Lip}(F)$.

Corollary 3.7. Let $E$ and $F$ be compact metric spaces, and let us assume that the set $\operatorname{Iso}\left(\left(\operatorname{Lip}(E),\|\cdot\|_{L}\right),\left(\operatorname{Lip}(F),\|\cdot\|_{L}\right)\right)$ is canonical. Then every 2-local $\operatorname{Iso}\left(\left(\operatorname{Lip}(E),\|\cdot\|_{L}\right),\left(\operatorname{Lip}(F),\|\cdot\|_{L}\right)\right)$-map $\Delta$ from $\operatorname{Lip}(E)$ to $\operatorname{Lip}(F)$ is a linear isometric map and there exist a closed subset $F_{0} \subset$ $F$, a Lipschitz map $\varphi: F_{0} \rightarrow E$ with $L(\varphi) \leq \max \{1, \operatorname{diam}(E)\}$, and $\tau \in \mathbb{T}$ such that

$$
\Delta(f)(s)=\tau f(\varphi(s)), \quad \forall f \in \operatorname{Lip}(E), \forall s \in F_{0} .
$$

Proof: Let $\Delta: \operatorname{Lip}(E) \rightarrow \operatorname{Lip}(F)$ be a 2-local isometry. Since $\Delta$ is a weak-2-local isometry, we deduce from Theorem 3.5 that $\Delta$ is linear. $\Delta$ being a 2-local isometry implies that $\Delta$ is a linear isometry. Namely, given $f, g \in \operatorname{Lip}(E)$ there exists a surjective linear isometry $T_{f, g}: \operatorname{Lip}(E) \rightarrow \operatorname{Lip}(F)$ such that $\Delta(f)=T_{f, g}(f)$ and $\Delta(g)=T_{f, g}(g)$. Therefore we have $\|\Delta(f)-\Delta(g)\|=\left\|T_{f, g}(f)-T_{f, g}(g)\right\|=\|f-g\|$, which shows that $\Delta$ is a linear isometry. 
The hypotheses also show that $\Delta\left(1_{E}\right)=\tau_{1_{E}}$ is a constant unimodular function. The desired conclusion follows from Theorem 2.4 in $[\mathbf{2 7}]$ and the facts that $\operatorname{Iso}\left(\left(\operatorname{Lip}(E),\|\cdot\|_{L}\right),\left(\operatorname{Lip}(F),\|\cdot\|_{L}\right)\right)$ is canonical, and $\Delta$ is a local isometry.

We can also obtain some other interesting consequences derived from Theorem 3.5. The following corollaries complement the conclusions in $[\mathbf{2 8}]$.

Corollary 3.8. Let $K$ be a compact metric space. Then every 2-local isometry $\Delta:\left(\operatorname{Lip}(K),\|\cdot\|_{s}\right) \rightarrow\left(\operatorname{Lip}(K),\|\cdot\|_{s}\right)$ is a surjective isometry.

Proof: By Theorem 3.5 every 2-local isometry $\Delta:\left(\operatorname{Lip}(K),\|\cdot\|_{s}\right) \rightarrow$ $\left(\operatorname{Lip}(K),\|\cdot\|_{s}\right)$ is a linear local isometry, and thus $\Delta$ is a local linear isometry. Now, applying Theorem 2.3 in $[\mathbf{2 6}]$ we derive that $\Delta$ is a surjective linear isometry.

Corollary 3.9. Let $K$ be a compact metric space. Suppose $K$ is connected with diameter at most 1 (or satisfies certain separation property to guarantee that $\operatorname{Iso}\left(\operatorname{Lip}(K),\|\cdot\|_{L}\right)$ is canonical). Then every 2-local isometry $\Delta:\left(\operatorname{Lip}(K),\|\cdot\|_{L}\right) \rightarrow\left(\operatorname{Lip}(K),\|\cdot\|_{L}\right)$ is a surjective isometry.

Proof: Let $\Delta:\left(\operatorname{Lip}(K),\|\cdot\|_{L}\right) \rightarrow\left(\operatorname{Lip}(K),\|\cdot\|_{L}\right)$ be a 2-local isometry. Theorem 3.5 implies that $\Delta$ is linear. Therefore, $\Delta$ is a linear isometry.

Although Theorem 2.3 in $[\mathbf{2 6}]$ is only stated for the norm $\|\cdot\|_{s}$, the rest of our arguments owe too much to the original proof by JiménezVargas, Morales Campoy, and Villegas-Vallecillos in [26], we include a brief argument for completeness reasons.

Since $\Delta$ is a linear 2-local isometry and $\operatorname{Iso}\left(\operatorname{Lip}(K),\|\cdot\|_{L}\right)$ is canonical, we conclude that $\Delta(1)=\tau_{1} \in \mathbb{T}$ is a constant function.

By Theorem 2.5 there exist a unimodular $\tau \in \operatorname{Lip}(K)$ and an algebra homomorphism $\psi: \operatorname{Lip}(K) \rightarrow \operatorname{Lip}(K)$ such that

$$
\Delta(f)=\tau \psi(f), \quad \forall f \in \operatorname{Lip}(K) .
$$

For each $s \in K$, the mapping $\delta_{s} \circ \psi: \operatorname{Lip}(K) \rightarrow \mathbb{C}$ is a non-zero multiplicative functional. In fact, $\tau_{1}=\Delta(1)=\tau \psi(1)$, and hence $1=\left|\tau_{1}\right|=|\tau(s) \psi(1)(s)|=|\psi(1)(s)|$, for all $s$ in $K$.

Since non-zero multiplicative linear functionals on $\left(\operatorname{Lip}(K),\|\cdot\|_{L}\right)$ and on $\left(\operatorname{Lip}(K),\|\cdot\|_{s}\right)$ are evaluation maps at a (unique) point in $K$ (see [45, Theorem 4.3.6]), then there exists a unique $\varphi(s) \in K$ such that $\delta_{s} \circ \psi=\delta_{\varphi(s)}$. We have thus defined a function $\varphi: K \rightarrow K$ satisfying

$$
\delta_{s} \circ \psi(f)=\delta_{\varphi(s)}(f), \quad \forall f \in \operatorname{Lip}(E) .
$$


Following the ideas in $[\mathbf{2 6}$, p. 199] we prove that $\varphi$ is injective. Namely, suppose that $\varphi(x)=\varphi(y)$, and let us take $h \in \operatorname{Lip}(K)$ such that $h^{-1}(\{0\})=\{\varphi(x)\}$. By the assumptions, there exist $\tau_{h} \in \mathbb{T}$ and a surjective isometry $\varphi_{h}: K \rightarrow K$ such that

$$
\Delta(h)(s)=\tau_{h} h\left(\varphi_{h}(s)\right), \quad \forall s \in K .
$$

Consequently,

$$
\tau_{h} h\left(\varphi_{h}(x)\right)=\Delta(h)(x)=\tau(x) \psi(h)(x)=\tau(x) h(\varphi(x))=0,
$$

and

$$
\tau_{h} h\left(\varphi_{h}(y)\right)=\Delta(h)(y)=\tau(y) \psi(h)(y)=\tau(y) h(\varphi(y))=0,
$$

which guarantees that $\varphi_{h}(x)=\varphi(x)=\varphi_{h}(y)$, and thus $x=y$.

We shall next check, following [26, proof of Theorem 2.3], that $\varphi$ is an isometry. Let us take $x \neq y$ in $K$, and $k \in \operatorname{Lip}(K)$ defined by $k(z):=$ $\frac{d(z, \varphi(x))}{d(z, \varphi(x))+d(z, \varphi(y))}$. Clearly, $k^{-1}(\{0\})=\{\varphi(x)\}$ and $k^{-1}(\{1\})=\{\varphi(y)\}$. By hypothesis, there exist $\tau_{k} \in \mathbb{T}$ and a surjective isometry $\varphi_{k}: K \rightarrow K$ such that

$$
\tau_{k} k\left(\varphi_{k}(x)\right)=\Delta(k)(x)=\tau(x) \psi(k)(x)=\tau(x) k(\varphi(x))=0,
$$

and

$$
\tau_{k} k\left(\varphi_{k}(y)\right)=\Delta(k)(y)=\tau(y) \psi(k)(y)=\tau(y) k(\varphi(y))=\tau(y) .
$$

We deduce from the properties of $k$ that $\varphi_{k}(x)=\varphi(x)$ and $\varphi_{k}(y)=\varphi(y)$. Since $\varphi_{k}$ is an isometry we get $d(\varphi(x), \varphi(x))=d\left(\varphi_{k}(x), \varphi_{k}(y)\right)=d(x, y)$ as desired.

Finally, since $\varphi: K \rightarrow K$ is an isometry on a compact metric space, Lemma 2.1 in $[\mathbf{2 6}]$ implies that $\varphi$ is surjective. Therefore the identity

$$
\Delta(f)(s)=\tau(s) \psi(f)(s)=\tau(s) f(\varphi(s))
$$

holds for all $f \in \operatorname{Lip}(K)$ and all $s \in K$. In particular, $\mathbb{T} \ni \tau_{1}=\Delta(1)=\tau$ is a constant function.

Back to the setting of uniform algebras, we can now combine our spherical variant of the Kowalski-Słodkowski theorem (see Proposition 3.2) with [17, Corollary 2.3.16] to study weak-2-local isometries.

Theorem 3.10. Let $A$ be a uniform algebra, let $Q$ be a compact Hausdorff space, and suppose that $B$ is a norm closed subalgebra of $C(Q)$ containing the constant functions. Then every weak-2-local isometry (respectively, every weak-2-local (algebraic) isomorphism) $\Delta: A \rightarrow B$ is a linear map.

Proof: Let $\Delta: A \rightarrow B$ be a weak-2-local isometry. We have already commented that $\Delta$ is homogeneous (see [12, Lemma 2.1]). If we fix an arbitrary $s \in Q$, the mapping $\delta_{s} \circ \Delta: A \rightarrow \mathbb{C}$ satisfies the hypothesis 
of Proposition 3.2. Indeed, since $\Delta$ is a weak-2-local isometry, given $a, b \in A$, by [17, Corollary 2.3.16], there exists an algebra isomorphism $\pi_{a, b, s}: A \rightarrow B$ and a unimodular $h_{a, b, s} \in B$ such that

$$
\delta_{s} \circ \Delta(a)=h_{a, b, s}(s) \pi_{a, b, s}(a)(s) \text {, and } \delta_{s} \circ \Delta(b)=h_{a, b, s}(s) \pi_{a, b, s}(b)(s) \text {. }
$$

Therefore

$$
\delta_{s} \circ \Delta(a)-\delta_{s} \circ \Delta(b)=h_{a, b, s}(s) \pi_{a, b, s}(a-b)(s) \in \mathbb{T} \sigma(a-b),
$$

as desired. Proposition 3.2 assures that $\delta_{s} \circ \Delta$ is linear. Therefore $\Delta$ is linear by the arbitrariness of $s$.

The statement concerning isomorphisms is a clear consequence of the fact that every isomorphism between $A$ and $B$ is an isometry.

Since 2-local isomorphisms and 2-local isometries between uniform algebras are a weak-2-local isometries, Theorem 3.10 provides a positive answer to Problems 1.1 and 1.2.

Corollary 3.11. Let $A$ and $B$ be uniform algebras. Then every 2-local isometry (respectively, every 2-local (algebraic) isomorphism) $\Delta: A \rightarrow B$ is a linear map.

Furthermore, if every local isometry (respectively, every 2-local (algebraic) isomorphism) from $A$ into $B$ is a surjective isometry (respectively, an isomorphism), then every 2-local isometry (respectively, every 2-local isomorphism) $\Delta: A \rightarrow B$ is a surjective linear isometry (respectively, an isomorphism).

Acknowledgements. L. Li was partly supported by NSF of China project no. 11301285. A. M. Peralta was partially supported by the Spanish Ministry of Economy and Competitiveness and European Regional Development Fund project no. MTM2014-58984-P and Junta de Andalucía grant FQM375. L. Wang was partly supported by NSF of China Grants No. 11371222, 11871303, and 11671133. Y.-S. Wang was partly supported by Taiwan MOST 104-2115-M-005-001-MY2.

Most of the results presented in this note were obtained during a visit of A. M. Peralta at the School of Mathematical Sciences in Nankai University. He would like to thank the first author and the Department of Mathematics for the hospitality during his stay.

The authors are very grateful to the anonymous referee for an exhaustive report containing many valuable suggestions to improve the final version of this paper.

\section{References}

[1] H. Al-Halees and R. J. Fleming, On 2-local isometries on continuous vector-valued function spaces, J. Math. Anal. Appl. 354(1) (2009), 70-77. DOI: $10.1016 / \mathrm{j} \cdot$ jmaa. 2008.12.023. 
[2] Sh. Ayupov and K. Kudaybergenov, 2-local derivations and automorphisms on B(H), J. Math. Anal. Appl. 395(1) (2012), 15-18. DOI: 110.1016/j.jmaa. 2012.04 .064 .

[3] Sh. Ayupov and K. Kudaybergenov, 2-local derivations on von Neumann algebras, Positivity 19(3) (2015), 445-455. DOI : 10.1007/s11117-014-0307-3.

[4] Sh. Ayupov, K. Kudaybergenov, and A. M. Peralta, A survey on local and 2-local derivations on $\mathrm{C}^{*}$ - and von Neumann algebras, in: "Topics in Functional Analysis and Algebra", Contemp. Math. 672, Amer. Math. Soc., Providence, RI, 2016, pp. 73-126. DOI : 10.1090/conm/672/13462.

[5] R. P. BOAs, JR., "Entire Functions", Academic Press Inc., New York, 1954.

[6] F. F. Bonsall and J. Duncan, "Complete Normed Algebras", Ergebnisse der Mathematik und ihrer Grenzgebiete 80, Springer-Verlag, New York-Heidelberg, 1973. DOI : $10.1007 / 978-3-642-65669-9$.

[7] M. J. Burgos, J. C. Cabello, and A. M. Peralta, Weak-local triple derivations on $\mathrm{C}^{*}$-algebras and JB*-triples, Linear Algebra Appl. 506 (2016), 614-627. DOI : 10.1016/j.laa.2016.06.042.

[8] M. Burgos, F. J. Fernández-Polo, J. J. Garcés, and A. M. Peralta, Local triple derivations on $C^{*}$-algebras, Comm. Algebra 42(3) (2014), 1276-1286. DOI : $10.1080 / 00927872.2012 .737191$.

[9] M. Burgos, F. J. Fernández-Polo, J. J. Garcés, and A. M. Peralta, A Kowalski-Słodkowski theorem for 2-local *-homomorphisms on von Neumann algebras, Rev. R. Acad. Cienc. Exactas Fís. Nat. Ser. A Math. RACSAM 109(2) (2015), 551-568. DOI : 10.1007/s13398-014-0200-8.

[10] M. J. Burgos, F. J. Fernández-Polo, J. J. Garcés, and A. M. Peralta, 2-local triple homomorphisms on von Neumann algebras and $\mathrm{JBW}^{*}$-triples, $J$. Math. Anal. Appl. 426(1) (2015), 43-63. DOI : 10.1016/j.jmaa.2014.12.058.

[11] M. Burgos, F. J. Fernández-Polo, and A. M. Peralta, Local triple derivations on C*-algebras and JB*-triples, Bull. Lond. Math. Soc. 46(4) (2014), 709-724. DOI : $10.1112 / \mathrm{blms} / \mathrm{bdu} 024$.

[12] J. C. Cabello and A. M. Peralta, Weak-2-local symmetric maps on C*-algebras, Linear Algebra Appl. 494 (2016), 32-43. DOI : 10.1016/j .laa.2015.12.024.

[13] J. C. Cabello and A. M. Peralta, On a generalized Šemrl's theorem for weak 2-local derivations on $B(H)$, Banach J. Math. Anal. 11(2) (2017), 382-397. DOI : $10.1215 / 17358787-0000009 x$.

[14] F. Cabello Sánchez and L. Molnár, Reflexivity of the isometry group of some classical spaces, Rev. Mat. Iberoamericana 18(2) (2002), 409-430. DOI: 10.4171/RMI/324.

[15] A. B. A. Essaleh, A. M. Peralta, and M. I. Ramírez, Weak-local derivations and homomorphisms on $C^{*}$-algebras, Linear Multilinear Algebra 64(2) (2016), 169-186. DOI : 10.1080/03081087.2015.1028320.

[16] A. B. A. Essaleh, A. M. Peralta, and M. I. Ramírez, Corrigendum: Weaklocal derivations and homomorphisms on $\mathrm{C}^{*}$-algebras, Linear Multilinear Algebra 64(5) (2016), 1009-1010. DOI : 10.1080/03081087.2016.1142171.

[17] R. J. Fleming and J. E. Jamison, "Isometries on Banach Spaces: Function Spaces", Chapman \& Hall/CRC Monographs and Surveys in Pure and Applied Mathematics 129, Chapman \& Hall/CRC, Boca Raton, FL, 2003.

[18] A. Fošner, 2-local Jordan automorphisms on operator algebras, Studia Math. 209(3) (2012), 235-246. DOI : 10.4064/sm209-3-3.

[19] A. Fošner, 2-local mappings on algebras with involution, Ann. Funct. Anal. 5(1) (2014), 63-69. DOI : 10.15352/afa/1391614570. 
[20] T. W. Gamelin, "Uniform Algebras", AMS Chelsea Publishing Series 311, Amer. Math. Soc., Providence, RI, 2005.

[21] A. M. Gleason, A characterization of maximal ideals, J. Analyse Math. 19(1) (1967), 171-172. DOI : $10.1007 /$ BF02788714.

[22] M. GYőRY, 2-local isometries of $C_{0}(X)$, Acta Sci. Math. (Szeged) 67(3-4) (2001), 735-746.

[23] O. Hatori, T. Miura, H. Oka, and H. Takagi, 2-local isometries and 2local automorphisms on uniform algebras, Int. Math. Forum 2(49-52) (2007), 2491-2502. DOI : 10.12988/imf .2007.07219.

[24] O. Hatori AND S. Oi, Isometries on Banach algebras of vector-valued maps, Acta Sci. Math. (Szeged) 84(1-2) (2018), 151-183. DOI : 10.14232/actasm-017558-6.

[25] K. Jarosz And V. D. Pathak, Isometries between function spaces, Trans. Amer. Math. Soc. 305(1) (1988), 193-206. DOI : 10.2307/2001048.

[26] A. Jiménez-Vargas, A. Morales Campoy, and M. Villegas-Vallecillos, Algebraic reflexivity of the isometry group of some spaces of Lipschitz functions, $J$. Math. Anal. Appl. 366(1) (2010), 195-201. DOI: 10.1016/j.jmaa.2010.01.034.

[27] A. JimÉnez-VARgas and M. Villegas-Vallecillos, Into linear isometries between spaces of Lipschitz functions, Houston J. Math. 34(4) (2008), 1165-1184.

[28] A. JimÉnez-VARgas and M. Villegas-Vallecillos, 2-local isometries on spaces of Lipschitz functions, Canad. Math. Bull. 54(4) (2011), 680-692. DOI : 10.4153/ CMB-2011-025-5.

[29] B. E. Johnson, Local derivations on $C^{*}$-algebras are derivations, Trans. Amer. Math. Soc. 353(1) (2001), 313-325. DOI : 10.1090/S0002-9947-00-02688-X.

[30] E. Jordá And A. M. Peralta, Stability of derivations under weak-2-local continuous perturbations, Aequationes Math. 91(1) (2017), 99-114. DOI : 10.1007/ s00010-016-0438-7.

[31] R. V. KADISON, Local derivations, J. Algebra 130(2) (1990), 494-509. DOI: 10.1016/0021-8693(90)90095-6.

[32] J.-P. Kahane AND W. ŻELAZKo, A characterization of maximal ideals in commutative Banach algebras, Studia Math. 29 (1968), 339-343. DOI : 10.4064/sm29-3-339-343.

[33] S. Kowalski And Z. SŁodkowski, A characterization of multiplicative linear functionals in Banach algebras, Studia Math. 67(3) (1980), 215-223. DOI: $10.4064 / \mathrm{sm}-67-3-215-223$.

[34] K. Kudaybergenov, T. Oikhberg, A. M. Peralta, and B. Russo, 2-local triple derivations on von Neumann algebras, Illinois J. Math. 58(4) (2014), $1055-1069$.

[35] D. R. LARson and A. R. Sourour, Local derivations and local automorphisms of $\mathcal{B}(X)$, in: "Operator Theory: Operator Algebras and Applications", Part 2 (Durham, NH, 1988), Proc. Sympos. Pure Math. 51, Amer. Math. Soc., Providence, RI, 1990, pp. 187-194. DOI : 10.1090/pspum/051.2/1077437.

[36] M. Mackey, Local derivations on Jordan triples, Bull. Lond. Math. Soc. 45(4) (2013), 811-824. DOI : 10.1112/blms/bdt007.

[37] P. Mankiewicz, On the differentiability of Lipschitz mappings in Fréchet spaces, Studia Math. 45 (1973), 15-29. DOI : 10.4064/sm-45-1-15-29.

[38] M. Niazi and A. M. Peralta, Weak-2-local *-derivations on $B(H)$ are linear *-derivations, Linear Algebra Appl. 487 (2015), 276-300. DOI: 10.1016/j.laa. 2015.09 .028$.

[39] M. Niazi and A. M. Peralta, Weak-2-local derivations on $\mathbb{M}_{n}$, Filomat 31(6) (2017), 1687-1708. DOI : 10.2298/FIL1706687N. 
[40] N. V. Rao and A. K. Roy, Linear isometries of some function spaces, Pacific J. Math. 38 (1971), 177-192. DOI : 10.2140/pjm.1971.38.177.

[41] A. K. Roy, Extreme points and linear isometries of the Banach space of Lipschitz functions, Canad. J. Math. 20 (1968), 1150-1164. DOI: 10.4153/CJM-1968109-9.

[42] P. Šemrl, Local automorphisms and derivations on $\mathcal{B}(H)$, Proc. Amer. Math. Soc. 125(9) (1997), 2677-2680. DOI : 10.1090/S0002-9939-97-04073-2.

[43] M. H. Vasavada, Closed ideals and linear isometries of certain function spaces, Ph.D Thesis, University of Wisconsin (1969).

[44] N. Weaver, Isometries of noncompact Lipschitz spaces, Canad. Math. Bull. 38(2) (1995), 242-249. DOI : 10.4153/CMB-1995-035-3.

[45] N. Weaver, "Lipschitz Algebras", World Scientific Publishing Co., Inc., River Edge, NJ, 1999. DOI : 10.1142/4100.

[46] W. ŻELAZKo, A characterization of multiplicative linear functionals in complex Banach algebras, Studia Math. 30 (1968), 83-85. DOI : 10.4064/sm-30-1-83-85.

L. Li

School of Mathematical Sciences and LPMC, Nankai University, Tianjin 300071, China

E-mail address: leilee@nankai.edu.cn

Antonio M. Peralta

Departamento de Análisis Matemático, Facultad de Ciencias, Universidad de Granada, 18071 Granada, Spain

E-mail address: aperalta@ugr.es

Liguang Wang

School of Mathematical Sciences, Qufu Normal University, Qufu 273165, China

E-mail address: wangliguang0510@163.com

Ya-Shu Wang

Department of Applied Mathematics, National Chung Hsing University, Taichung 402, Taiwan

E-mail address: yashu@nchu.edu.tw

Primera versió rebuda el 10 de maig de 2017, darrera versió rebuda el 17 de novembre de 2017. 